\title{
Single-ventricle anatomy predicts delayed microstructural brain development
}

\author{
Viyeka Sethi', Sarah Tabbutt ${ }^{1}$, Anastasia Dimitropoulos ${ }^{2}$, Kevin C. Harris² ${ }^{2}$ Vann Chau ${ }^{2}$, Kenneth Poskitt ${ }^{3}$, Andrew Campbell ${ }^{4}$, \\ Anthony Azakie ${ }^{5}$, Duan $\mathrm{Xu}^{6}$, Anthony J. Barkovich ${ }^{6}$, Steven P. Miller ${ }^{1,2,7}$ and Patrick S. McQuillen ${ }^{1}$
}

BACKGROUND: Term newborns with congenital heart disease (CHD) show delayed brain development as early as the third trimester, especially in single-ventricle physiology (SVP). Mechanisms causing delayed brain development in CHD are uncertain but may include impaired fetal brain blood flow. Our objective was to determine if cardiac anatomy associated with obstruction to antegrade flow in the ascending aorta is predictive of delayed brain development as measured by diffusion tensor imaging and magnetic resonance spectroscopic (MRS) imaging.

METHODS: Echocardiograms from 36 term newborns with SVP were reviewed for the presence of aortic atresia and the diameter of the ascending aorta. Quantitative magnetic resonance imaging parameters measuring brain microstructural (fractional anisotropy (FA) and average diffusivity (Dav)) or metabolic development (N-acetylaspartate (NAA) and lactate/ choline (Lac/Cho)) were recorded.

RESULTS: Increasing NAA/Cho and white matter (WM) FA, and decreasing Dav and Lac/Cho characterize normal brain development. Consistent with the hypothesis that delayed brain development is related to impaired brain perfusion, smaller ascending aortic diameter and aortic atresia were associated with higher Dav and lower WM FA. Echocardiogram variables were not associated with brain metabolic measures.

CONCLUSIONS: These observations support the hypothesis that obstruction to fetal cerebral blood flow impairs brain microstructural development.

A dverse neurodevelopmental outcome is commonly noted in children with many forms of congenital heart disease (CHD), and the origins are pleiotropic. Acquired perioperative neurologic injury in the form of focal and diffuse white matter injury (WMI) and small infarcts can be identified in the majority of infants with serious CHD requiring neonatal surgery (1-4). Therefore, most efforts to improve neurodevelopmental outcome have focused on intraoperative care and cardiopulmonary bypass (5-9). Risk factors and mechanisms for these acquired brain injuries are incompletely described but are speculated to include hypoxia, ischemia, inflammation, and embolism $(10,11)$. Reported risk factors for postoperative brain injury on magnetic resonance imaging (MRI) include sustained and low regional cerebral oxygen saturation, low diastolic blood pressure, and brain immaturity $(1,12,13)$. Increasing evidence, however, suggests that clinical and radiological neurologic abnormalities are present at birth (14) and may begin during fetal life (15).

MRI techniques have been developed to quantitatively measure aspects of brain development (16). For instance, diffusion tensor imaging (DTI) measures the direction and magnitude of water movement. With increasing brain microstructural development, the magnitude of brain water diffusion decreases and directionality increases, particularly in the WM. Structural axonal connectivity is maximal around birth and decreases postnatally. Analogous changes occur in metabolic compounds that can be measured by magnetic resonance spectroscopy (MRS). MRS measures brain metabolites, some of which exhibit distinct changes with development including increase in $\mathrm{N}$-acetylaspartate (NAA) and decrease in lactate (Lac) (17). Use of these techniques, as well as measurement of brain volumes, has demonstrated that newborns with CHD have significant delays in brain development before surgery $(18,19)$. Onset of this delay in brain development occurs during the third trimester in utero (15). Delayed brain development could result from disruption of shared genetic or morphological developmental programs because many of the genes identified as causal for CHD also have important roles in brain development (20). A separate explanation arises from the observation that many forms of $\mathrm{CHD}$ affect fetal circulation to decrease brain oxygen or nutrient delivery, which might result in delayed brain development. Patients with hypoplastic left heart syndrome (HLHS), for example, have a spectrum of impairment to left-ventricular ejection, from complete aortic atresia to mild aortic stenosis, that will variably influence the usual preferential streaming of oxygen and nutrientrich placental blood antegrade through the aorta to the brain. This hypothesis suggests the testable prediction that postnatal

'Department of Pediatrics and Neurology, University of California, San Francisco Benioff Children's Hospital, San Francisco, California; ${ }^{2}$ Department of Pediatrics, University of British Columbia, British Columbia Children's Hospital, Vancouver, British Columbia, Canada; ${ }^{3}$ Department of Radiology, University of British Columbia, British Columbia Children's Hospital, Vancouver, British Columbia, Canada; ${ }^{4}$ Department of Surgery, University of British Columbia, British Columbia Children's Hospital, Vancouver, British Columbia, Canada; ${ }^{5}$ Department of Surgery, University of California, San Francisco Benioff Children's Hospital, San Francisco, California; ${ }^{6}$ Department of Radiology, University of California, San Francisco Benioff Children's Hospital, San Francisco, California; ${ }^{7}$ Current affiliation: Department of Paediatrics, The Hospital for Sick Children and the University of Toronto, Toronto, Ontario, Canada. Correspondence: Patrick S. McQuillen (mcquillp@peds.ucsf.edu) 
Table 1. Clinical characteristics

\begin{tabular}{lc}
\hline & $n=36$ \\
\cline { 2 - 2 } Variable & $\begin{array}{c}\text { Mean } \pm \text { SD or } \\
\text { median (range) }\end{array}$ \\
\hline Male (\%) & $18(52)$ \\
Birth weight (g) & $3,185 \pm 472$ \\
Birth head circumference (cm) & $34 \pm 1.4$ \\
Birth length (cm) & $49 \pm 6$ \\
Apgar score at 5 min & $9(5-9)$ \\
SNAP-PE & $15(10-59)$ \\
Gestational age at birth (wk) & $38.8 \pm 1$ \\
Gestational age at MRI (wk) & $39.4 \pm 1$ \\
MRI day & $5(1-13)$ \\
Preoperative lowest $\mathrm{O}_{2}$ saturation (\%) & $77 \pm 11$ \\
\hline
\end{tabular}

MRI, magnetic resonance imaging; SNAP-PE, Score for Neonatal Acute PhysiologyPerinatal Extension, ref. 27.

Table 2. Cardiac characteristics

\begin{tabular}{llcc}
\hline $\begin{array}{l}\text { Cardiac } \\
\text { physiology }\end{array}$ & $\begin{array}{c}\text { Cardiac anatomy } \\
(n)\end{array}$ & $\begin{array}{c}\text { Aortic } \\
\text { diameter, mm } \\
(\text { mean } \pm \text { SD) }\end{array}$ & $\begin{array}{c}\text { Head } \\
\text { circumference, } \\
\text { cm (mean } \pm \text { SD) }\end{array}$ \\
\hline $\begin{array}{l}\text { SV without } \\
\text { arch obstruction } \\
(n=3)\end{array}$ & $\begin{array}{l}\text { SV with PA (2) } \\
\text { Unbalanced }\end{array}$ & $8.2 \pm 1.5$ & 37.5 \\
AVC (1) & 8.1 & 34 \\
$\begin{array}{l}\text { SV with arch } \\
\text { obstruction } \\
(n=33)\end{array}$ & HLHS with aortic & $2.3 \pm 0.6$ & $34.3 \pm 1.6$ \\
& atresia (16) & & \\
& HLHS (6) & $4.9 \pm 0.9$ & $34.7 \pm 1.3$ \\
& TGA, SV (4) & $5 \pm 1$ & $33.7 \pm 0.3$ \\
& Unbalanced & $5.2 \pm 1.2$ & $34.6 \pm 1.4$ \\
& AVC (4) & & \\
& DORV (3) & $6.5 \pm 0.9$ & $34.3 \pm 1.1$
\end{tabular}

AVC, atrioventricular canal; DORV, double-outlet right ventricle; HLHS, hypoplastic left heart syndrome; PA, pulmonary atresia; SV, single ventricle; TGA, transposition of great arteries.

echocardiographic parameters in patients with single-ventricle physiology (SVP), suggestive of increased obstruction to antegrade flow to the head and neck vessels, should be associated with greater magnitude of delayed brain development on quantitative MRI. Specifically, we hypothesize that increasing ascending aortic diameter or absence of aortic atresia, both indicative of more fetal antegrade flow, will be associated with less delay of microstructural and metabolic brain development.

\section{RESULTS}

\section{Patient Characteristics}

A total of 36 term infants (median gestational age: $38.8 \mathrm{wk}$ ), 18 male and 18 female, were identified with SVP. Their demographic data and postpartum characteristics are summarized in Table 1. Most of the infants were either prenatally diagnosed $(n=22)$ or were diagnosed before developing shock or cardiac arrest due to closure of the ductus arteriosus. Therefore, the Score for Neonatal Acute Physiology-Perinatal Extension, a validated illness severity and mortality risk score used in newborn intensive
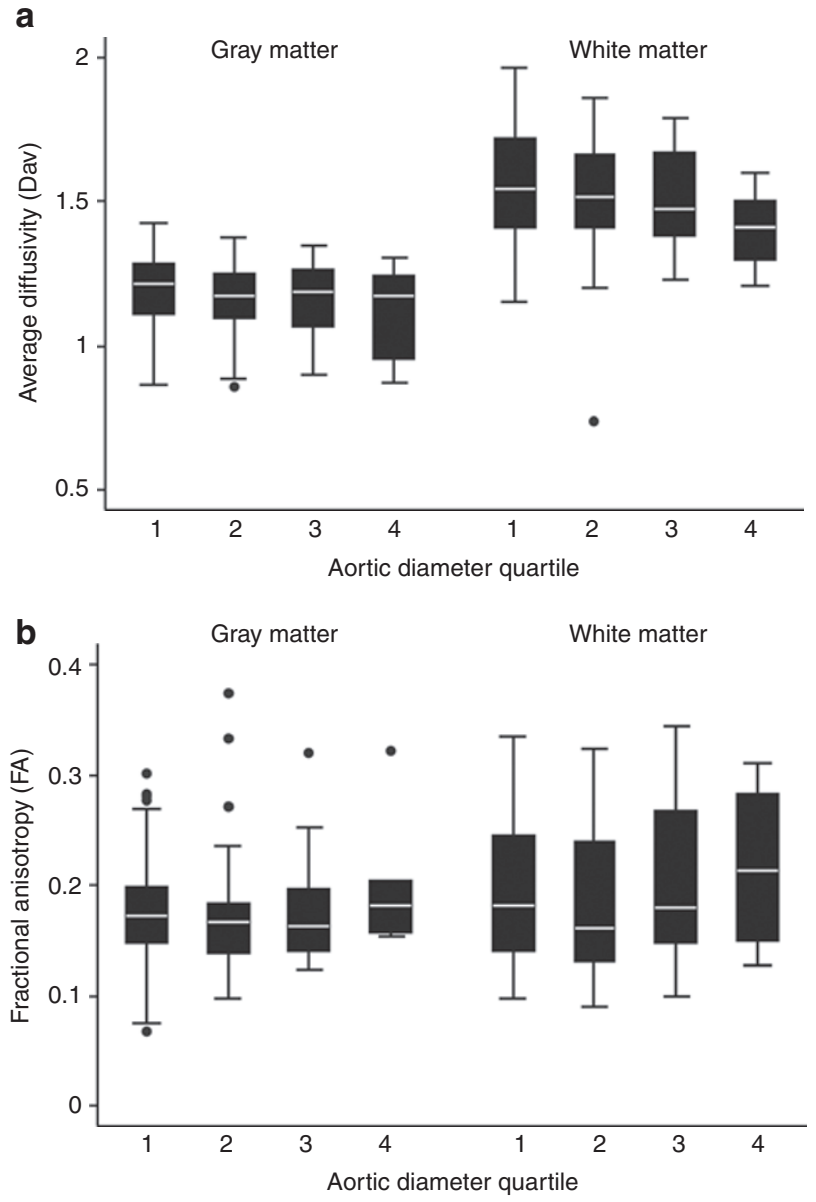

Figure 1. Relationship between aortic diameter and magnetic resonance diffusion measurements. (a) Box and whisker plots of average diffusivity (Dav) and (b) fractional anisotropy (FA) by arch quartile. Gray (basal ganglia, thalamus, calcarine cortex) and white matter (optic radiations and posterior, perirolandic, and frontal white matter) regions of interest are averaged and plotted against quartiles of aortic diameter $(1=0-2.5 \mathrm{~mm} ; 2=2.6-5 \mathrm{~mm}$; $3=5.1-7.5 \mathrm{~mm} ; 4=7.6-10 \mathrm{~mm}$ ). Each plot consists of the median, white line in the middle of the black box; the 25th and 75th percentiles, ends of the black box; and the 5th and 95th percentile, ends of the whiskers. Outliers are indicated by filled circles.

care patients (21), was not excessively high (median score: 15 ). The lowest daily preoperative oxygen saturation noted in the bedside flow sheet was $77 \% \pm 11 \%$, indicating an expected degree of hypoxia. The majority of patients included had a diagnosis of HLHS $(n=21)$ or variants of HLHS with aortic arch obstruction and required surgical palliation in the form of a Norwood procedure (Table 2). A smaller number of subjects $(n=3)$ did not have arch obstruction and received various surgical palliations. A total of 16 patients (43.2\%) with aortic atresia were in the cohort.

\section{Preoperative Brain Injury}

Most patients had MRI performed in the first postnatal week (median day of life for preoperative MRI $=5 \mathrm{~d}$; interquartile range: 3-6 d). Brain injury was observed on the preoperative MRI in 13 of 36 infants (36.1\%), a similar percentage to that reported in other studies with larger populations and no restrictions on the type of $\mathrm{CHD}(1,2,22,23)$. Brain injuries 
included mild WMI in 4 patients (11.1\%), moderate in 4 patients $(11.1 \%)$, and severe WMI in 2 of 36 newborns (5.5\%). Focal strokes were noted in 6 of 36 patients (16.7\%). All strokes were small, less than one-third of the vascular territory. Strokes occurred in the middle $(n=5)$ or posterior $(n=1)$ cerebral artery territories. Intraventricular hemorrhages occurred in five patients (13.5\%), grade I in one patient $(2.7 \%)$ and grade II in four patients $(10.8 \%)$. No cases of global hypoxic-ischemic injury were observed in this cohort.

Ascending Aorta Diameter and Aortic Atresia Are Associated With The mean average diffusivity (Dav) for gray matter across all seven regions of interest (ROIs) for our cohort was $1.17 \pm 0.012 \mathrm{~mm}^{2} \times 10^{-3} / \mathrm{s}$ and for WM was $1.52 \pm 0.016 \mathrm{~mm}^{2}$ $\times 10^{-3} / \mathrm{s}$. The mean fractional anisotropy (FA) in the WM regions, obtained in a similar manner, was $0.19 \pm 0.004$. This is a comparably higher Dav and lower WM FA than previously published values for a cohort of healthy infants at term (24). Similarly, our cohort had comparably lower mean

Table 3. Results of echocardiographic and quantitative diffusion and spectroscopy variables

\begin{tabular}{|c|c|c|c|c|}
\hline Predictor variable & $\begin{array}{l}\text { Outcome } \\
\text { variable }\end{array}$ & Coefficient & $P$ value & $95 \% \mathrm{Cl}$ \\
\hline \multirow{4}{*}{$\begin{array}{l}\text { Ascending } \\
\text { aortic diameter }\end{array}$} & Dav & -16.25 & $<0.001$ & -25.21 to -7.30 \\
\hline & $\begin{array}{l}\text { FA white } \\
\text { matter }\end{array}$ & 5.35 & $<0.001$ & $2.40-8.30$ \\
\hline & Eigenvector 1 & -18.58 & 0.005 & -33.66 to -3.50 \\
\hline & $\begin{array}{l}\text { Eigenvector } \\
2-3 \text { (mean) }\end{array}$ & -25.55 & $<0.001$ & $\begin{array}{l}-38.92 \text { to } \\
-12.19\end{array}$ \\
\hline \multirow[t]{4}{*}{ Aortic atresia } & Dav & 72.65 & $<0.001$ & $37.68-107.61$ \\
\hline & $\begin{array}{l}\text { FA white } \\
\text { matter }\end{array}$ & -18.51 & 0.009 & -32.30 to -4.72 \\
\hline & Eigenvector 1 & 90.36 & 0.005 & $27.70-153.04$ \\
\hline & $\begin{array}{l}\text { Eigenvector } \\
2-3 \text { (mean) }\end{array}$ & 108.54 & $<0.001$ & 48.33-168.76 \\
\hline
\end{tabular}

$\mathrm{Cl}$, confidence interval; Dav, average diffusivity; FA, fractional anisotropy.

a

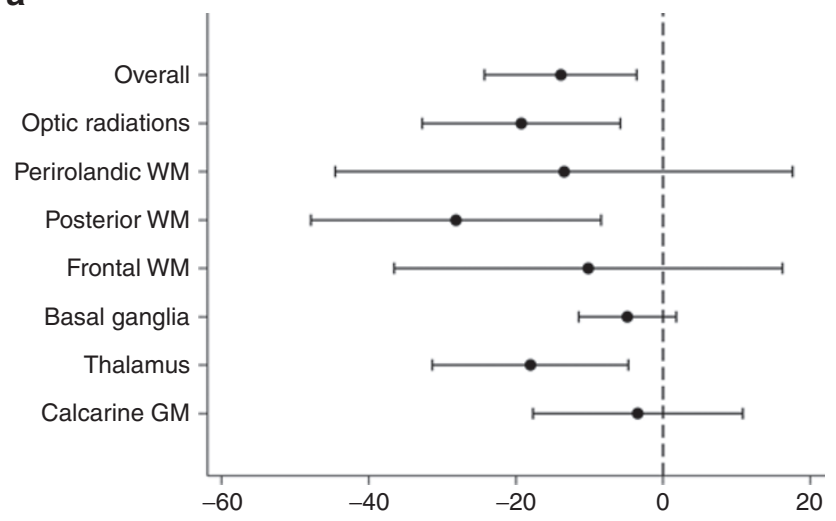

$\mathrm{NAA} /$ choline $(\mathrm{Cho})$ ratios $(\mathrm{NAA} / \mathrm{Cho}=0.566 \pm 0.01)$ and higher mean Lac/Cho ratios (Lac/Cho $=0.142 \pm 0.01)$ across all seven ROIs than previously published values for healthy neonates (24).

Aortic diameter for a normal $3.5-\mathrm{kg}$ neonate is $9.3 \pm 0.9 \mathrm{~mm}$ (25). Aortic diameters for each CHD group and anatomical diagnosis are summarized in Table 2. To explore the effect of increasing aortic diameter on quantitative magnetic resonance measurements, we plotted the diffusion variables, Dav and FA $\mathrm{WM}$, as well as the spectroscopic variables, NAA/Cho and Lac/Cho, against quartiles of ascending aortic diameter. We summarized the raw data by gray matter and WM ROIs. These plots demonstrate that Dav decreases with increasing ascending aortic diameter quartile (Figure 1a). Similarly, we have seen an increase in FA across each ascending aortic quartile or increasing diameter (Figure 1b). NAA/Cho and Lac/Cho ratios did not show a consistent relationship with arch diameter quartile (data not shown).

We then tested the association of predictor and outcome variables using a linear regression for repeated measures, corrected for gestational age at the time of the MRI (Table 3). We found a significant inverse relationship between aortic diameter and Dav, in that, as the ascending aortic diameter increased, the mean Dav across all ROIs decreased $(P<0.001$; coefficient: -16.3 ; $95 \%$ confidence interval (CI): -25.2 to -7.3 ). The converse association was seen with WM FA and ascending aortic diameter. As the ascending aortic diameter increased, mean WM FA increased across all ROIs $(P<0.001$; coefficient: 5.4 ; 95\% CI: 2.4-8.3). The relationship was significant for both axial (eigenvector 1) and radial (mean eigenvector 2, 3; Table 3) measures of diffusivity, although the radial measure was more strongly associated. Subjects with aortic atresia exhibit the smallest aortic diameters (mean \pm SD: $2.3 \pm 0.6 \mathrm{~mm}$ ). Given the absence of antegrade flow into the ascending aorta, we repeated this analysis dividing subjects into groups of atresia and quartiles of aortic diameter for the remaining subjects. Using linear regression, identical results were found including a significant inverse relationship between group and mean Dav ( $P<0.001$; coefficient: -18.5 ; $95 \% \mathrm{CI}$ : -32.7 to -4.4$)$, as

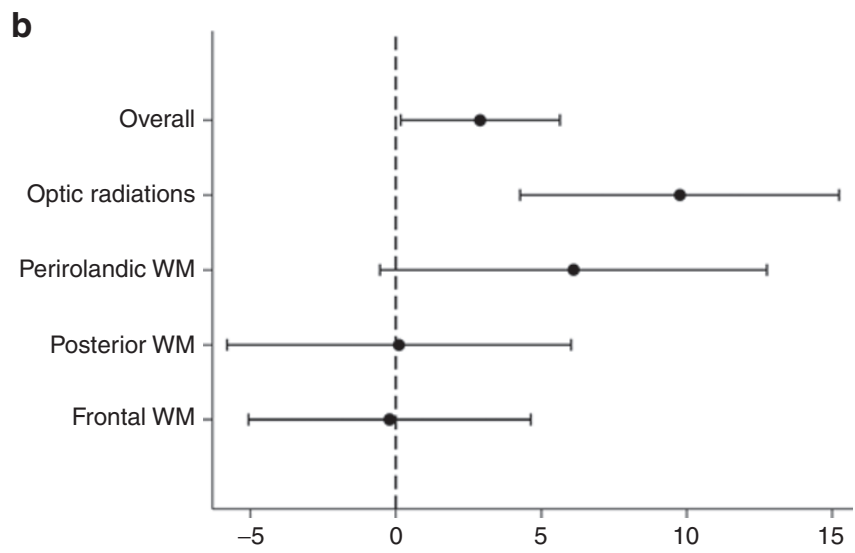

Figure 2. Results of the regression analyses overall and by regions of interest for the association of (a) average diffusivity and (b) fractional anisotropy

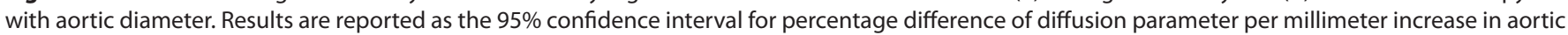
diameter. GM, gray matter; WM, white matter. 


\section{Articles | Sethi etal.}

well as WM FA ( $P<0.001$; coefficient: 6.0 ; 95\% CI: 0.9-11.1). The spectroscopic variables show less consistent relationships with increasing ascending aortic diameter. The diameter of the ascending aorta did not predict a significant change in the spectroscopic variables NAA/Cho $(P=0.814$; coefficient: 0.002 ; $95 \%$ CI: -0.01 to 0.02$)$ or $\mathrm{Lac} / \mathrm{Cho}(P=0.145$; coefficient: $0.008 ; 95 \%$ CI: -0.00 to 0.02 ). This lack of association did not change when analyzing subjects categorically.

We also used linear regression for repeated measures while correcting for the gestational age at the time of scanning to analyze the relationship between the presence of aortic atresia and microstructural and metabolic brain delay. We found a statistically significant direct relationship between higher Dav in neonates with aortic atresia $(P<0.001$; coefficient: $72.6 ; 95 \% \mathrm{CI}$ : 37.6-107.6), consistent with microstructural brain immaturity in the setting of aortic atresia. Similarly, there was a significant inverse relationship between WM FA and aortic atresia $(P=$ 0.009; coefficient: -18.5 ; $95 \%$ CI: -32.3 to -4.7$)$. Moreover, the relationship was significant for both axial and radial diffusivity, with a stronger association for the radial measure (Table 3 ). The presence of aortic atresia did not significantly predict the differences in NAA/Cho $(P=0.157$; coefficient: 0.032 ; $95 \%$ CI: -0.01 to 0.08$)$ or $\mathrm{Lac} / \mathrm{Cho}(P=0.245$; coefficient: -0.019 ; $95 \%$ CI: -0.05 to 0.01 ). This relationship held when the analysis was limited to either WM or gray matter alone.

As described above, we saw significant relationships between ascending aortic diameter or aortic atresia and diffusion variables in a pattern suggesting delayed microstructural brain development. To explore whether this overall effect was similar across each ROI, we plotted the percentage change in Dav and FA across individual ROIs in comparison with the overall effect (Figure 2a,b). We found the associations did not differ meaningfully across the ROIs for both Dav and FA, suggesting a widespread brain association.

Ascending Aortic Diameter and Aortic Atresia Do Not Predict WMI Preoperative WMI is observed frequently in newborns with SVP. We tested the relationship between aortic diameter and/or aortic atresia and preoperative WMI. Using logistic regression, we found no significant relationship between smaller ascending aortic diameter and the presence of WMI ( $P=0.656$; coefficient: $-0.08 ; 95 \%$ CI: -0.44 to 0.27 ). Using a $\chi^{2}$ test, we found that the presence of aortic atresia was not significantly associated with the presence of WMI $(P=0.360)$.

\section{DISCUSSION}

By comparing measurements of cardiac anatomy with quantitative magnetic resonance parameters of brain development, we have determined that aortic diameter and the presence of aortic atresia are significantly associated with microstructural WM development in newborns with SVP. Specifically, we found that aortic diameter and the presence of aortic atresia predict Dav and WM FA. Increasing aortic diameter was associated with lower Dav and higher WM FA, a pattern associated with more advanced brain maturity. During cardiovascular development, increasing blood flow is thought to be associated with increased size and development of many cardiovascular structures, including chambers, valves, and major vessels. Smaller aortic diameter or aortic atresia is suggestive of a pattern of restricted left-ventricular ejection and diminished flow in the ascending aorta to the head and neck vessels. Overall, this pattern is consistent with the hypothesis that diminished antegrade fetal cerebral blood flow and oxygen and nutrient delivery leads to delayed brain development.

Changes in brain magnetic resonance diffusion and MRS measurements over normal fetal and postnatal development provide a simplistic model for analyzing alterations in microstructural and metabolic brain development in CHD. Further support for this paradigm comes from alternative approaches utilizing macroscopic features of brain development including size, volume, formation of gyri and sulci, myelination, and disappearance of germinal matrix (18). Using both tools, newborns with CHD appear to be $\sim 1$ mo immature in comparison with normal newborns. Relevance for this concept is further supported by observations that certain features (e.g., brain volumes) can normalize over time following the repair of $\mathrm{CHD}$ (26). Clearly, many features of brain development, including neuronal circuit and synapse formation, cannot be assessed by current MRI tools, and thus, brain structural and functional abnormalities may exist but not be detected by MRI.

In normal fetal circulation, oxygenated blood with an oxygen saturation $\sim 80-85 \%$ is returned through the umbilical vein and ductus venosus via the hepatic circulation. Umbilical venous blood (oxygen saturation: $\sim 73 \%$ ) is preferentially shunted across the foramen ovale to the left atrium to supply the developing fetal brain. In CHD, there is a spectrum of abnormal blood flow and mixing that may lead to diminished brain oxygen and nutrient delivery. The most severely affected are those with HLHS. In HLHS, there is an impairment to leftventricular ejection, resulting in reversal of flow across the foramen ovale and complete mixing of oxygenated blood in the atrium. Left-sided obstruction may further diminish the antegrade flow of this less saturated blood, and in the most severe cases, the developing fetal brain is supplied in a retrograde manner via the ductus arteriosus.

Infants with CHD have smaller head volumes as compared with infants with structurally normal hearts, and this is particularly apparent in infants with HLHS (27). Head growth slows in fetuses with HLHS during the third trimester (28). This might be related to restriction of flow through the aorta. In a retrospective study of 129 infants with HLHS, ascending and transverse aorta measurements and presence of aortic valve atresia/stenosis were tested as predictors of microcephaly within that population (29). Among these, only a smaller ascending aortic diameter significantly predicted the presence of microcephaly $(P=0.034)$. There was no association between microcephaly and aortic atresia. Additional factors associated with microcephaly identified in the study were interactions between anatomical restrictions to flow, cerebral vascular resistance, and cerebral blood flow.

The importance of antegrade flow is illustrated by recent evidence of its role in delayed brain development in third-trimester 
fetuses. In a prospective study, brain volume and metabolism were compared in 55 infants with CHD and 50 controls (15). Infants with CHD had smaller brain volumes and lower NAA/ Cho ratios, indicating abnormal metabolic brain development. Infants with HLHS and transposition of the great arteries had the lowest NAA/Cho ratios and mild elevations of Lac/ Cho. Moreover, the lack of flow through the ascending aorta was a significant independent predictor of a lower NAA/Cho ratio. The measures of antegrade flow and cerebral blood flow in these studies capture a fixed moment in time, whereas the contributions to brain development are based on complex dynamic interactions among the cerebral vasculature, intracardiac flow, and ventricular output.

Of note, no significant associations were found between cardiac anatomical predictors and spectroscopy outcomes (NAA, Lac) in our cohort. A lack of association persisted even if spectroscopic variables were analyzed separately for WM and gray matter regions. This is consistent with our previous findings in a mixed population (SVP and transposition of the great arteries), in which Lac was not significantly elevated relative to controls (19). One possible explanation for the lack of associations for NAA might relate to the prevalence of glial pathology observed in fetuses and newborns with CHD. NAA is predominantly a neuronal biomarker. The neuropathological sequela of WMI is a failure of normal myelination (30) resulting from the destruction of oligodendrocyte progenitors and a maturation arrest of the oligodendrocyte precursor pool (31). Of note, increases in WM FA coincide with immature oligodendrocyte progression and maturation (32). WMI is also associated with an increase of reactive astrocytes and activated microglia in both premature newborns (33) and fetuses with CHD (28). The onset of pathology in fetuses with SVP overlaps a peak in the proliferation of vulnerable oligodendrocyte progenitors (34). Finally, in our study, changes in WM FA were driven by larger changes in radial diffusion (eigenvector 2,3), rather than longitudinal (eigenvector 1) diffusion, consistent with a disturbance of myelination.

Our hypothesis that a static measurement of postnatal aortic diameter reflects the dynamic range of fetal cerebral blood flow is overly simplistic. An exception to this paradigm occurs in the condition of fetal aortic stenosis, in which fetal left heart structures are normal sized at diagnosis yet the systemic ventricle becomes progressively dysfunctional. A subset of these patients progresses to HLHS. In an effort to better identify candidates for fetal balloon aortic valvuloplasty, studies have identified important predictors of that transformation. Among those, the presence of retrograde flow in the transverse arch was found to predict the progression to HLHS with 100\% sensitivity and specificity (35). The heterogeneous onset and severity of left-sided obstructive lesions, their impact on leftventricular function, and the downstream effects on antegrade cerebral blood flow are difficult to quantify using current imaging technologies. More sensitive, dynamic measurements of fetal cerebral blood flow are needed in the setting of adequate ventricular mass and outflow tract but reduced ventricular function. Measurement of retrograde blood flow in the aorta might have improved our ability to identify this subset of patients and improved the association with magnetic resonance measures of metabolic brain development in our cohort. However, in our population, almost all patients had large patent ductus arteriosus, which interfered with the assessment of retrograde flow in the transverse arch via Doppler flow.

We found significant relationships for the diffusion variables (Dav and WM FA) and the ascending aortic diameter, as well as for aortic atresia. Examining the regional variability of these observations, Dav is higher with smaller aortic diameter across all ROIs. However, with WM FA, the effect is more heterogeneous, with the largest changes in the optic radiations and perirolandic WM. One explanation for this observation relates to the timing of WM myelination. WM maturation occurs at variable rates and follows a set pattern (36-38). The usual course follows myelination in the cerebral peduncles and pons at birth, followed by the posterior limb of the internal capsule. The optic radiations and pyramidal tract motor fibers are among the first cortical regions to myelinate after birth and are actively myelinating at the time of the preoperative scanning. Other regions, including the anterior limb of the internal capsule, the genu of the corpus callosum, and finally the anterior, posterior, and parietal WM, mature at later ages.

This study carries important limitations including the lack of comparison to a normal control group without CHD. Despite this limitation, we demonstrate a significant association between ascending aortic diameter and the presence of aortic atresia and MRI measures of microstructural brain development (Dav and WM FA). Specifically, we found that smaller ascending aorta diameters or the presence of aortic atresia predict a higher Dav and a lower WM FA in SVP. Our data implicate an important role for impaired antegrade cerebral blood flow in delayed microstructural brain development in newborns with CHD. Future studies will be necessary to determine if fetal interventions to improve cerebral blood flow also restore brain development and improve outcomes.

\section{METHODS}

\section{Patients}

Newborns with SVP who were born at or transferred to the British Columbia Children's Hospital in Vancouver (University of British Columbia (UBC)) and the University of California Benioff Children's Hospital San Francisco (UCSF) were studied in an ongoing prospective cohort study. SVP was defined as the absence of one of the two functioning ventricles where heart function in series would be incompatible with life and palliative surgical intervention is required. Enrollment of patients began in 2001 at UCSF and in 2007 at UBC and continued through 2011 at both centers. Exclusion criteria included gestational age at birth $<36 \mathrm{wk}$ and suspected congenital infection or suspected/ confirmed genetic or malformation syndrome. A total of 147 patients were consented and enrolled between the two centers. From the overall cohort, all 36 infants with SVP were included in this analysis (34 newborns at UCSF). Newborns were enrolled after receiving written informed consent from their parents. Once informed consent was obtained, brain MRI was performed before and after cardiac surgery.

Institutional committees on human research at both UCSF and UBC approved the study protocol.

\section{Echocardiography}

Preoperative echocardiograms were performed on all patients as part of routine clinical care. Measurements were carried out by a single pediatric cardiologist at each center (UCSF: S.T. and UBC: K.C.H.) 
who was blinded to the neuroimaging results. Presence of aortic atresia and the size of the ascending aorta above the sinotubular junction were determined according to the American Society of Echocardiography chamber quantification guidelines (39) as described; see ref. 40 for a diagram of the position of aortic diameter measurement. Aortic atresia was defined as the absence of antegrade flow.

\section{MRI Studies}

Preoperative MRI studies were performed as soon as the baby could safely be transported to the MRI scanner with the use of a specialized MRI-compatible isolette with a neonatal head coil. Studies at UCSF were performed with pharmacologic sedation, as needed, on a 1.5 Tesla system (GE Healthcare Signa Echo-speed; GE Healthcare, Buckinghamshire, UK) and included: 4-mm-thickness T1-weighted sagittal and axial spin echo, 4-mm-thickness dual-echo T2-weighted spin echo, 1.5-mm-thickness coronal or sagittal volumetric three-dimensional gradient echo with radiofrequency spoiling images, and a diffusion tensor sequence (repetition time: $7000 \mathrm{~ms}$; echo time: $99.5 \mathrm{~ms}$; 3 -mm section thickness; no gap; three repetitions per image, with $18 \times 36 \mathrm{~cm}$ field of view and $128 \times 256$ acquisition matrix), acquiring axial images through the whole brain with an in-plane resolution of $1.4 \times 1.4 \mathrm{~mm}^{2}$. We acquired seven images per axial section, including a T2-weighted reference image ( $b=0$ $\left.\mathrm{s} / \mathrm{mm}^{2}\right)$ and 6-15 diffusion-weighted images $\left(b=700 \mathrm{~s} / \mathrm{mm}^{2}\right)$ in noncollinear gradient directions. At UBC, MRI studies were carried out without pharmacologic sedation on a Siemens 1.5 Tesla Avanto (Siemens AG, Healthcare Sector, Erlangen, Germany) using VB 13A software (Siemens AG, Healthcare Sector) and included imaging sequences comparable to those of the UCSF (23). No adverse events occurred during this protocol. A neuroradiologist reviewed each MRI for focal, multifocal, or global changes as described previously (20). WMI was classified as follows: mild ( $1-3$ foci each $<2 \mathrm{~mm}$ ), moderate ( $>3$ foci of $>2 \mathrm{~mm}$ ), or severe ( $>5 \%$ of WM volume).

\section{Three-Dimensional MRS Imaging}

MRS used point-resolved spectroscopy pulse sequence technique using a repetition time of $1 \mathrm{~s}$ and an echo time of $144 \mathrm{~ms}$ to allow for multivoxel imaging as described previously $(19,41)$. The spectra were analyzed using automated routines developed by our group with voxels centered on seven listed anatomic regions in the WM and gray matter bilaterally: (i) basal ganglia, (ii) thalamus, (iii) optic radiation, (iv) calcarine region, (v) corticospinal tracts, (vi) posterior WM and (vii) frontal WM; see ref. 19 for exact position of voxel locations. Each voxel was reviewed by a neuroradiologist (UCSF: A.J.B. and UBC: K. P.) to ensure consistency of voxel placement and an adequate signal intensity to noise ratio. Peak-area ratios of Lac/Cho, Lac/NAA, and NAA/Cho were calculated. The values from the left and right hemispheres were averaged, and a mean value was used for analysis. NAA is a neuronal marker that increases as the brain matures. Lac is a marker of anaerobic metabolism that is almost undetectable in brain parenchyma at birth in normal healthy term infants. NAA/Cho and Lac/Cho ratios have been previously shown to increase (NAA/Cho) or decrease (Lac/Cho) during brain development $(17,42)$.

\section{Diffusion Tensor Imaging}

DTI was performed using a sequence optimized at each site for neonatal brain imaging (described in refs. 19,41, and DTI data have been analyzed across both the centers in other neonatal studies, ref. 43). The diffusion tensor in this situation is an ellipsoid, the size and form of which manifests the direction and amount of free water diffusion, as described by maximum eigenvalues and corresponding eigenvectors of the tensor. Dav describes the magnitude of water motion during the period; it is examined by each excitation during the DTI sequence. FA describes the degree of directionality of water motion; a value closer to zero describes isotropic diffusion (water molecules that move equally in all directions), whereas a value close to one describes diffusion predominantly in one direction. WM is relatively anisotropic as compared with gray matter. Dav was calculated for voxels in the same seven anatomic regions as MRS imaging, and FA was calculated for the WM regions.

\section{Statistical Analyses}

We compared our predictor variables (aortic arch diameter and presence of aortic atresia) with the unadjusted mean values for ratios of
NAA and Lac to Cho, Dav, and FA of WM and gray matter. We used linear regression for repeated measures for this comparison while adjusting for gestational age at the time of the MRI. These models each included an interaction term for site (UCSF or UBC) by ROI. The interaction term for site by ROI allows for the MRS imaging and DTI values in each region to vary by site (UCSF or UBC) because study subjects were imaged in different MRI scanners at two medical centers.

Logistic regression was also used to evaluate whether ascending aortic diameter was predictive of WMI and $\chi^{2}$ analysis was used to evaluate whether aortic atresia was predictive of WMI. All variables were analyzed using Stata software, version 12 (Stata, College Station, TX), using a $P$ value $<0.05$ to determine statistical significance.

\section{STATEMENT OF FINANCIAL SUPPORT}

This study was supported by the National Institute of Neurological Disorders and Stroke at the US National Institutes of Health (grant 1R01NS063876), Canadian Institutes of Health Research, and the March of Dimes (grant 6-FY2009-303). S.P.M. is currently Bloorview Children's Hospital Chair in Pediatric Neuroscience and was supported by a Tier 2 Canadian Research Chair in Neonatal Neuroscience, and Michael Smith Foundation for Health Research Scholar award.

Disclosure: This study and study investigators have no disclosures, including no financial ties to products in the study or potential/perceived conflicts of interest.

\section{REFERENCES}

1. Andropoulos DB, Hunter JV, Nelson DP, et al. Brain immaturity is associated with brain injury before and after neonatal cardiac surgery with highflow bypass and cerebral oxygenation monitoring. J Thorac Cardiovasc Surg 2010;139:543-56.

2. Mahle WT, Tavani F, Zimmerman RA, et al. An MRI study of neurological injury before and after congenital heart surgery. Circulation 2002;106:12 Suppl 1:I109-14.

3. McQuillen PS, Hamrick SE, Perez MJ, et al. Balloon atrial septostomy is associated with preoperative stroke in neonates with transposition of the great arteries. Circulation 2006;113:280-5.

4. McQuillen PS, Barkovich AJ, Hamrick SE, et al. Temporal and anatomic risk profile of brain injury with neonatal repair of congenital heart defects. Stroke 2007;38:Suppl 2:736-41.

5. Clancy RR, McGaurn SA, Goin JE, et al. Allopurinol neurocardiac protection trial in infants undergoing heart surgery using deep hypothermic circulatory arrest. Pediatrics 2001;108:61-70.

6. du Plessis AJ, Jonas RA, Wypij D, et al. Perioperative effects of alpha-stat versus $\mathrm{pH}$-stat strategies for deep hypothermic cardiopulmonary bypass in infants. J Thorac Cardiovasc Surg 1997;114:991-1000; discussion 1000-1.

7. Goldberg CS, Bove EL, Devaney EJ, et al. A randomized clinical trial of regional cerebral perfusion versus deep hypothermic circulatory arrest: outcomes for infants with functional single ventricle. J Thorac Cardiovasc Surg 2007;133:880-7.

8. Jonas RA, Wypij D, Roth SJ, et al. The influence of hemodilution on outcome after hypothermic cardiopulmonary bypass: results of a randomized trial in infants. J Thorac Cardiovasc Surg 2003;126:1765-74.

9. NewburgerJW,JonasRA,WernovskyG,etal.Acomparisonoftheperioperative neurologic effects of hypothermic circulatory arrest versus low-flow cardiopulmonary bypass in infant heart surgery. N Engl J Med 1993;329:1057-64.

10. du Plessis AJ. Neurologic complications of cardiac disease in the newborn. Clin Perinatol 1997;24:807-26.

11. Wernovsky G. Current insights regarding neurological and developmental abnormalities in children and young adults with complex congenital cardiac disease. Cardiol Young 2006;16:Suppl 1:92-104.

12. Dent CL, Spaeth JP, Jones BV, et al. Brain magnetic resonance imaging abnormalities after the Norwood procedure using regional cerebral perfusion. J Thorac Cardiovasc Surg 2006;131:190-7.

13. Galli KK, Zimmerman RA, Jarvik GP, et al. Periventricular leukomalacia is common after neonatal cardiac surgery. J Thorac Cardiovasc Surg 2004;127:692-704.

14. Limperopoulos C, Majnemer A, Shevell MI, Rosenblatt B, Rohlicek C, Tchervenkov C. Neurologic status of newborns with congenital heart defects before open heart surgery. Pediatrics 1999;103:402-8. 
15. Limperopoulos C, Tworetzky W, McElhinney DB, et al. Brain volume and metabolism in fetuses with congenital heart disease: evaluation with quantitative magnetic resonance imaging and spectroscopy. Circulation 2010;121:26-33.

16. Hüppi PS, Dubois J. Diffusion tensor imaging of brain development. Semin Fetal Neonatal Med 2006;11:489-97.

17. Kreis R, Ernst T, Ross BD. Development of the human brain: in vivo quantification of metabolite and water content with proton magnetic resonance spectroscopy. Magn Reson Med 1993;30:424-37.

18. Licht DJ, Shera DM, Clancy RR, et al. Brain maturation is delayed in infants with complex congenital heart defects. J Thorac Cardiovasc Surg 2009;137:529-36; discussion 536-7.

19. Miller SP, McQuillen PS, Hamrick S, et al. Abnormal brain development in newborns with congenital heart disease. N Engl J Med 2007;357:1928-38.

20. McQuillen PS, Goff DA, Licht DJ. Effects of congenital heart disease on brain development. Prog Pediatr Cardiol 2010;29:79-85.

21. Richardson DK, Phibbs CS, Gray JE, McCormick MC, Workman-Daniels K, Goldmann DA. Birth weight and illness severity: independent predictors of neonatal mortality. Pediatrics 1993;91:969-75.

22. Beca J, Gunn J, Coleman L, et al. Pre-operative brain injury in newborn infants with transposition of the great arteries occurs at rates similar to other complex congenital heart disease and is not related to balloon atrial septostomy. J Am Coll Cardiol 2009;53:1807-11.

23. Block AJ, McQuillen PS, Chau V, et al. Clinically silent preoperative brain injuries do not worsen with surgery in neonates with congenital heart disease. J Thorac Cardiovasc Surg 2010;140:550-7.

24. Bartha AI, Yap KR, Miller SP, et al. The normal neonatal brain: MR imaging, diffusion tensor imaging, and 3D MR spectroscopy in healthy term neonates. AJNR Am J Neuroradiol 2007;28:1015-21.

25. Kampmann C, Wiethoff CM, Wenzel A, et al. Normal values of M mode echocardiographic measurements of more than 2000 healthy infants and children in central Europe. Heart 2000;83:667-72.

26. Ibuki K, Watanabe K, Yoshimura N, et al. The improvement of hypoxia correlates with neuroanatomic and developmental outcomes: comparison of midterm outcomes in infants with transposition of the great arteries or single-ventricle physiology. J Thorac Cardiovasc Surg 2012;143:1077-85.

27. Rosenthal GL. Patterns of prenatal growth among infants with cardiovascular malformations: possible fetal hemodynamic effects. Am J Epidemiol 1996;143:505-13.

28. Hinton RB, Andelfinger G, Sekar P, et al. Prenatal head growth and white matter injury in hypoplastic left heart syndrome. Pediatr Res 2008;64:364-9.

29. Shillingford AJ, Ittenbach RF, Marino BS, et al. Aortic morphometry and microcephaly in hypoplastic left heart syndrome. Cardiol Young 2007;17:189-95.

30. Buser JR, Maire J, Riddle A, et al. Arrested preoligodendrocyte maturation contributes to myelination failure in premature infants. Ann Neurol 2012;71:93-109.
31. Segovia KN, McClure M, Moravec M, et al. Arrested oligodendrocyte lineage maturation in chronic perinatal white matter injury. Ann Neurol 2008;63:520-30.

32. Drobyshevsky A, Song SK, Gamkrelidze G, et al. Developmental changes in diffusion anisotropy coincide with immature oligodendrocyte progression and maturation of compound action potential. J Neurosci 2005;25: 5988-97.

33. Back SA. Perinatal white matter injury: the changing spectrum of pathology and emerging insights into pathogenetic mechanisms. Ment Retard Dev Disabil Res Rev 2006;12:129-40.

34. Back SA, Luo NL, Borenstein NS, Volpe JJ, Kinney HC. Arrested oligodendrocyte lineage progression during human cerebral white matter development: dissociation between the timing of progenitor differentiation and myelinogenesis. J Neuropathol Exp Neurol 2002;61: 197-211.

35. Mäkikallio K, McElhinney DB, Levine JC, et al. Fetal aortic valve stenosis and the evolution of hypoplastic left heart syndrome: patient selection for fetal intervention. Circulation 2006;113:1401-5.

36. Barkovich AJ, Kjos BO, Jackson DE Jr, Norman D. Normal maturation of the neonatal and infant brain: MR imaging at 1.5 T. Radiology 1988;166(1 Pt 1):173-80.

37. Brody BA, Kinney HC, Kloman AS, Gilles FH. Sequence of central nervous system myelination in human infancy. I. An autopsy study of myelination. J Neuropathol Exp Neurol 1987;46:283-301.

38. Kinney HC, Brody BA, Kloman AS, Gilles FH. Sequence of central nervous system myelination in human infancy. II. Patterns of myelination in autopsied infants. J Neuropathol Exp Neurol 1988;47:217-34.

39. Lang RM, Bierig M, Devereux RB, et al.; Chamber Quantification Writing Group; American Society of Echocardiography's Guidelines and Standards Committee; European Association of Echocardiography. Recommendations for chamber quantification: a report from the American Society of Echocardiography's Guidelines and Standards Committee and the Chamber Quantification Writing Group, developed in conjunction with the European Association of Echocardiography, a branch of the European Society of Cardiology. J Am Soc Echocardiogr 2005;18: 1440-63.

40. Gautier M, Detaint D, Fermanian C, et al. Nomograms for aortic root diameters in children using two-dimensional echocardiography. Am J Cardiol 2010;105:888-94.

41. Chau V, Poskitt KJ, McFadden DE, et al. Effect of chorioamnionitis on brain development and injury in premature newborns. Ann Neurol 2009;66:155-64.

42. Glenn OA. Normal development of the fetal brain by MRI. Semin Perinatol 2009;33:208-19.

43. Bonifacio SL, Glass HC, Chau V, et al. Extreme premature birth is not associated with impaired development of brain microstructure. J Pediatr 2010;157:726-32.e1. 